21 BVerfG, Urt. v. 04.05.2011 - 2 BvR 2365/09 u.a., NJW 2011, 1931 ff. = JZ 2011, 845 ff.

22 BVerfGE 109, 133, 167

23 BVerfG (Fn.21), Rn. $111-118$.

24 BVerfG (Fn.21), Rn. 125 und 126 unter Hinweis auf Bartsch (Fn. 6).

25 EGMR Urt. v. 17.12.2009 - 19359/04 (M. ./ Deutschland), Rn. $121 \mathrm{ff}$.

26 BVerfGE 109, 133, $167 \mathrm{ff}$.

27 BVerfG (Fn. 21), Rn. 93; zustimmend Hörnle, NStZ 2011, 488, 489; Böttcher, Forum Strafvollzug 2011, 281, $283 \mathrm{f}$.

28 BVerfG (Fn. 21), Rn. 172.

29 BVerfG (Fn. 21), Tenor III. 2. a) und Rn.173.

30 EGMR Urt. v. 17.12.2009 - 19359/04 (M. ./ Deutschland) Rn. 125, NJW 2010, 2495 ff. $=\mathrm{StV}$ 2010, 181 ff.; zur Konventionskonformität der Sicherungsverwahrung auch Urt. v. 21.10.2010 - 24478/03 (G. ./Deutschland) Rn. $45-54$.

31 Unter Hinweis auf MK-StGB/Radtke, 1. Aufl. 2003, Vor $\mathbb{S} \$ 38$ ff. Rn. 68.
32 BVerfG (Fn. 21), Rn. 104; ebenso bereits BVerfGE 109, 133,159.

33 BVerfG (Fn. 21) Rn. 129.

34 Ebenso Nußstein, StV 2011, 633; BMJ Pressemitteilung vom 22.9.2011; BMJ RefE (Fn. 20) Art. 316f II EGStGB; a.A. OLG Nürnberg, Beschl. v. 21.7.2011 - 15 W 1400, BeckRS 2011, 19213.

35 Frädrich/Pfäfflin, R\&P 2000, 95, 96 f.

36 Herpetz/Saß, Allgemeine Epidemiologie, Verlauf, Prognose, in: Herpetz/Saß (Hrsg), Persönlichkeitsstörungen, 2003, 177 - 182; ähnliche Ergebnisse in der internationalen Übersicht bei Nedopil, Forensische Psychiatrie, 3. Aufl. 2007, S. 188

37 Vgl. dazu Schöch, GA 2012, 14, 23 ff.

38 Dittmann, in: Nedopil (Hrsg.), Die Psychiatrie und das Recht - Abgrenzung und Brückenschlag, 2011, S. 131- 150; Müller, in Nedopil (Hrsg.) 201, 115-130; ähnlich auch Höffler/ Stadtland, StV 2012, 242 f.; Renzikowski, ZIS 2011, 531-543.

39 Schöch, GA 2012, 14, 27 ff.
40 Dazu Höffler/Stadtland StV 2012, 244.

41 Bundesministerium der Justiz, Referentenentwurf eines Gesetzes zur bundesrechtlichen Umsetzung des Abstandgebotes im Recht der Sicherungsverwahrung vom 9.11.2011, http:// www.der-paritaetische.de/fachinfos/artikel/ news/stellungnahme-zur-reform-der-sicherungsverwahrung/ abgerufen am 6.2.2012.

42 BMJ (Fn. 41), S. 14.

43 BMJ (Fn. 41), S. 18.

44 http://www.bmj.de/SharedDocs/Pressemitteilungen/DE/2011/20111109_Sicherungsverwahrung_verfassungsfest.html?nn=1930246.

45 Kaiser/Schöch, Strafvollzug, 5. Aufl. 2002, $\$ 10$ Rn. 74.

46 BVerfG (Fn. 21) Rn. 97.

47 Schöch, FS Roxin 2011, 1193, 1212 m.w.N.; Feltes/Alex, FS Schöch 2010, 750 f.; Kinzig, Die Legalbewährung gefährlicher Rückfalltäter, 2008, 196 ff.

\title{
Die Regelungen der Sicherungsverwahrung im Lichte des Bundesverfassungsgerichtsurteils vom 04.05.2011 in ihren Auswirkungen auf Psychiatrie und Psychotherapie
}

Jürgen L. Müller

Die Maßregel der Sicherungsverwahrung soll die Gesellschaft vor gefährlichen Straftätern schützen. Es ist die einschneidendste Sanktion des deutschen Strafrechts, die von den Betroffenen ein großes individuelles Sonderopfer abverlangt. Die schuldunabhängig, kriminalpräventiv verhängte Maßregel dient einerseits als ultima ratio dem Schutz der Gesellschaft, nimmt aber andererseits zugleich die Gesellschaft in die Pflicht, dieses Sonderopfer tunlichst nicht oder zumindest so wenig eingreifend wie möglich erforderlich werden zu lassen. Hierauf wurde seitens des Bundesverfassungsgerichts bereits hingewiesen. Ungeachtet dessen blieb die Wirkung des Verfassungsgerichts auf die Vollzugspraxis bislang gering. Mit den aktuellen Urteilen des Europäischen Gerichtshofs für Menschenrechte jedoch wurde der besondere Charakter dieses kriminalpräventiv abverlangten Freiheitsverzichts nochmals hervorgehoben und jetzt sehr nachhaltig. Für gefährlich erachtete Probanden mussten in die Freiheit entlassen werden, zum Teil rund um die Uhr polizeilich überwacht; besondere Betreuungssituationen wurden einzelfallbezogen geschaffen. Nicht zuletzt rückte zu diesem für erforder- lich erachteten Schutz der Gesellschaft sodann die Psychiatrie in den Fokus der Gesetzgebung, um die für besonders gefährlich erachteten Straftäter nunmehr mit dem Etikett einer psychischen Störung gelabelt verwahren zu können. Dabei besteht die Zielgruppe der Sicherungsverwahrung aber gerade aus hoch gefährlichen Straftätern, die bei Begehung ihrer Tat für schuldfähig erachtet wurden und bei denen eine erhebliche psychische Störung im Sinne der Eingangsmerkmale der $\mathbb{S} 20,21$ StGB gerade nicht festgestellt wurde. Dementsprechend wurde bei diesen Probanden die Unterbringung in einem psychiatrischen Krankenhaus gemäß \$63 StGB auch nicht angeordnet, stattdessen eine Haftstrafe mit anschließender Sicherungsverwahrung verhängt. Im Zuge der Neuregelung der Sicherungsverwahrung sowie insbesondere mit Inkrafttreten des Therapieunterbringungsgesetzes wurden die Grenzen zwischen Psychiatrie, Justizvollzug und Verwahrung erneut auf den Prüfstand gestellt. Im Folgenden werden die aktuelle Entwicklung im Kontext der Sicherung von hochgefährlichen Straftätern aus psychiatrischer Sicht und seitens der Fachgesellschaft reflektiert und die Auswirkungen der
Regelungen auf Psychiatrie und Psychotherapie diskutiert.

\section{Entwicklung}

Der beabsichtigte Ausnahmecharakter der Sicherungsverwahrung schlägt sich in den Jahren von 1980 bis 1995 in niedrigen Anordnungsraten und konstant niedrigen Zahlen nieder. 1995 befanden sich 183 Straftäter in Sicherungsverwahrung, 2010 waren es 524; zwischen 1990 und 1995 wurden nie mehr als 40 Personen im Jahr zur Sicherungsverwahrung verurteilt. Während noch in den 1990er Jahren die Sicherungsverwahrung so stark an Bedeutung verloren hatte, dass sie bereits als eine aussterbende Maßregel bezeichnet wurde, kam es in Folge der Tötungsdelikte an Nathalie und Kim 1998 zu einer Änderung des Strafgesetzbuches und zu wiederholten Verschärfungen und Ausweitungen der Maßregel und in der Folge zu einem deutlichen Anstieg der Verwahrtenzahlen. Seither wurde diese eigentlich als ultima ratio konzipierte Maßnahme zu einer inzwischen immer üblicheren Maßnahme. In den zurückliegenden 15 Jahren haben sich die Verwahrtenzahlen 
beinahe schon verdreifacht (s. dazu Schöch, in diesem Heft). Im selben Zeitraum wurde die Maßregel der Sicherungsverwahrung 12 Mal verändert (Pollähne, 2011). Dabei gerieten die Bestimmungen der Maßregel zunehmend in Konflikt mit der Rechtsprechung des Bundesverfassungsgerichts sowie mit den Regelungen der Europäischen Menschenrechtskommission (EMRK) bzw. der Rechtsprechung des Europäischen Gerichtshofs für Menschenrechte (EGMR):

1998 war die Höchstdauer der erstmaligen Sicherungsverwahrung aufgehoben worden. Seit diesem Zeitpunkt können als besonders gefährlich eingestufte Straftäter auf unbegrenzte Zeit verwahrt werden. Das Gesetz wurde auch rückwirkend auf diejenigen Straftäter angewandt, die sich bei Inkrafttreten des Gesetzes schon in der Sicherungsverwahrung befanden bzw. bei denen die Maßregel schon angeordnet worden war.

2004 wurde die nachträgliche Verlängerung der Sicherungsverwahrung vom Bundesverfassungsgericht als verfassungskonform beurteilt, da es sich bei der Sicherungsverwahrung um eine Maßregel und nicht um eine Strafe handele. Daher komme dem Rückwirkungsverbot, demzufolge keine Strafe rückwirkend verlängert oder nachträglich angeordnet werden darf, im Kontext der Sicherungsverwahrung keine Bedeutung zu.

Im Gegensatz zu dieser Position des Bundesverfassungsgerichts wertete der Europäische Gerichtshof für Menschenrechte (EGMR) im Urteil vom 17.12.2009 die Sicherungsverwahrung als Strafe, da ihr Vollzug sich nicht wesentlich von der Verbüßung einer Strafhaft unterscheidet und dementsprechend die nachträgliche Verlängerung der Sicherungsverwahrung gegen das Rückwirkungsverbot verstößt. Mit dem Urteil des Europäischen Gerichtshofs für Menschenrechte waren die Betroffenen zu entlassen. Im Januar 2011 rügte der Europäische Gerichtshof für Menschenrechte auch generell die nachträglich verhängte Sicherungsverwahrung von Straftätern in Deutschland und verurteilte die Bundesrepublik Deutschland zur Zahlung einer Entschädigung. Auf Grundlage des Urteils des EGMR vom 17.12.2009 und der Entscheidung vom Mai 2010, die Beschwerde der Bundesregierung nicht einmal zur Verhandlung zuzulassen, wurde innerhalb weniger Monate das Gesetz zur Neuordnung des Rechts der Sicherungsverwahrung und zu den begleitenden Regelungen umgesetzt. Dieses Gesetz, das auch das für Psychiatrie und Psychotherapie besonders relevante Therapieunterbrin- gungsgesetz (ThUG) umfasst, ist seit dem 01.01.2011 in Kraft getreten.

\section{Therapieunterbringungsgesetz}

$\mathrm{Zu}$ diesem Gesetz bezog die Deutsche Gesellschaft für Psychiatrie, Psychotherapie und Nervenheilkunde (DGPPN) in der Stellungnahme vom 10.02.2011 kritisch Position (Müller et al. 2011a). Insbesondere wurde kritisiert, dass

- das vorgelegte Gesetz rein inhaltlich die Kritik des EGMR- Urteils verfehlt,

das Gesetz zu einem Missbrauch der Psychiatrie führt, weil Kriminalität und Krankheit sowie dauerhafte Gefährlichkeit und psychische Krankheit gleichgesetzt werden,

- es nicht nachvollziehbar ist, davon auszugehen, dass psychotherapeutische Maß-nahmen die betroffenen Wiederholungstäter nach mehr als zehn Jahren Justizvollzug innerhalb von 18 oder 36 Monaten ungefährlich machen,

hochfrequente Begutachtungen in 18-monatigen Abständen die Hoffnung auf eine Entlassung nach „Heilung“ von der Gefährlichkeit schüren.

Dieses Gesetz formuliert den Begriff der „psychischen Störung“ als Voraussetzung einer Unterbringung nach dem ThUG. Dieser bis dahin im rechtlichen Kontext unbekannte und undefinierte Begriff wurde wegen der Einführung zum Zwecke der Unterbringung und wegen der Unbestimmtheit des Konstrukts heftig kritisiert. Die Etikettierung eines bis dahin als nicht psychisch krank im Rechtsinne eingestuften, aber hochgefährlichen Straftäters mit einer „psychischen Störung“, mit dem Zweck diesen weiter verwahren zu können, ist aus psychiatrischer Sicht ein Sündenfall, den die Fachgesellschaft deutlich kritisiert hat als einen Versuch, die Kriminalpolitik auf dem Rücken und zu Lasten psychiatrischer Patienten zu betreiben (Müller et al. 2011a). Verschiedene Auslegungen fordern für den hier eingeführten Begriff der „psychischen Störung “ als Eingangsmerkmal für eine Therapierungsunterbringung einen solchen Schweregrad, wie er in der Rechtsprechung für die Annahme zumindest einer erheblich beeinträchtigten Schuldfähigkeit definiert ist (hierzu: Koller 2012). Dies steht ganz im Einklang mit den Konzeptionen der etablierten Rechtsprechung und der forensisch psychiatrischen Beurteilungspraxis. Ein deutlich weiter auslegbares Verständnis des
Begriffs der „psychischen Störung “ legte jedoch das ThUG zugrunde und grenzte sich von den etablierten Konzepten der Schuldfähigkeitsbeurteilung $a b$, da diese zu eng seien. Auch das Bundesverfassungsgericht wies in einer Entscheidung vom 15. September 2011 (2 BvR 1516/11) darauf hin, dass bereits wiederkehrende aggressive Handlungen hinreichend sein können, eine psychische Störung im Sinne des ThUG zu begründen. Letztlich deutete der EGMR in einer Entscheidung vom 19. Januar 2012 über den Fall KRONFELDNER v. GERMANY (Application no. 21906/09) an, dass die Beeinträchtigung durch die „psychische Störung " rechtserheblich sein müsse (hierzu Koller, 2012). Dies ist wiederum ganz im Sinne der etablierten forensisch psychiatrischen Beurteilung. Mittlerweile hat es auf der Grundlage dieses Gesetzes erste Unterbringungen von Betroffenen in psychiatrischen Kliniken oder eigens hierfür vorbereiteten Institutionen gegeben. Gegen die Anwendung des ThUG und die unbestimmte Kategorie „psychische Störung“ richtet sich die aktuell anhängige Verfassungsbeschwerde eines im Saarland untergebrachten Straftäters. Es wird abzuwarten sein, wie sich das Bundesverfassungsgericht zur Erheblichkeit der psychischen Beeinträchtigung für die Annahme des Merkmals einer „psychischen Störung " im Sinne des ThUG positionieren wird.

\section{Urteil des Bundesverfassungsgerichts vom 04.05.2011}

Das Bundesverfassungsgericht beendete 2011, angesichts der EGMR Rechtsprechung und der wiederholten Urteile gegen die Bundesrepublik Deutschland, der aufgegeben wurde, die Untergebrachten freizulassen, die 1998 begonnene Serie der Ausweitung und Verschärfung der Maßregel der Sicherungsverwahrung. Mit Verkündung vom 4. Mai 2011 hat der Zweite Senat des Bundesverfassungsgerichts entschieden, dass alle Vorschriften des Strafgesetzbuches und des Jugendgerichtsgesetzes über die Anordnung und Dauer der Sicherungsverwahrung mit dem Freiheitsgrundrecht der Untergebrachten nicht vereinbar sind, weil sie den Anforderungen des verfassungsrechtlichen Abstandsgebots nicht genügen. Die Vorschriften zur nachträglichen Verlängerung der Sicherungsverwahrung über die frühere Zehnjahreshöchstfrist hinaus und zur nachträglichen Anordnung der Sicherungsverwahrung im Erwachsenenund Jugendstrafrecht verletzen zudem das 
rechtsstaatliche Vertrauensschutzgebot. Bis zum Inkrafttreten einer gesetzlichen Neuregelung, längstens bis zum 31. Mai 2013, hat das Bundesverfassungsgericht die weitere Anwendbarkeit der für verfassungswidrig erklärten Vorschriften angeordnet. In den Übergangsregelungen war die Fortdauer der Sicherungsverwahrung bei Altfällen allerdings nur noch möglich, wenn eine hochgradige Gefahr schwerster Gewalt- oder Sexualstraftaten aus konkreten Umständen in der Person oder dem Verhalten des Untergebrachten abzuleiten ist und dieser an einer psychischen Störung im Sinne von $\mathbb{} 1$ Absatz 1 Nr. 1 des Therapieunterbringungsgesetzes (ThUG) leidet. Damit griff auch das Bundesverfassungsgericht den heftig kritisierten Begriff der „psychischen Störung“ auf. Dennoch begrüßte die DGPPN (Müller et al. 2011b) die Entscheidung des Bundesverfassungsgerichts zur Sicherungsverwahrung, da es die Politik dazu zwingt, aus der Aneinanderreihung von immer neuen häufig anlassbezogenen Gesetzen und Ergänzungen, die auch für die beteiligten Juristen, Vollzugspraktiker und forensischen Psychiater kaum mehr überschaubar ist, ein einheitliches Konzept zu entwickeln, welches der Balance zwischen Sicherheitsbedürfnissen und Freiheitsrechten nach rechtsstaatlichen Prinzipien gerecht wird. Denn von der vom Bundesverfassungsgericht geforderten Neukonzeption der Regelungen der Sicherungsverwahrung ist die Psychiatrie und insbesondere die forensische Psychiatrie und Psychotherapie in hohem Maße betroffen, da nicht nur Begutachtungen als Grundlage der gerichtlichen Entscheidungen weiterhin erforderlich sein werden, sondern auch weil der Therapieanspruch der in der Sicherungsverwahrung Untergebrachten im Urteil des Bundesverfassungsgerichts besonders betont wird und damit die einzelnen Maßregeln näher aneinanderrücken (Müller et al. 2011b). Wegen der Verbindungen der Maßregeln (psychiatrischer Maßregelvollzug, Entziehungsbehandlung und Sicherungsverwahrung) und wegen des auch bei der Sicherungsverwahrung verankerten Anspruchs auf Behandlung und Resozialisierung müssen Indikation und Durchlässigkeit zwischen verschiedenen Unterbringungsformen geregelt werden, mit dem Ziel der optimalen Rehabilitation der jeweils Betroffenen. Da-

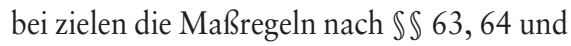
66 StGB sowie das ThUG auf unterschiedliche Tätergruppen. Inhaltlich formulierte die DGPPN dementsprechend konkrete Empfehlungen zur Neuregelung der Sicherungsverwahrung angesichts des Urteils des
Bundesverfassungsgerichts vom 04.04.2011 (Müller et al. 2011c). Im Einzelnen wurde postuliert,

den Begriff „psychische Störung“ zu ersetzen,

die Sozialtherapeutischen Einrichtungen (SothA) weiter auszugestalten und $\mathrm{zu}$ entwickeln, ein resozialisierungsförderndes Setting innerhalb der SothA zu etablieren, die Betroffenen durch die Gewährung von Lockerungen vorzubereiten und einen geeigneten Empfangsraum vor der Entlassung einzurichten und zu erproben,

die Verlegung psychisch gestörter Sicherungsverwahrter in Einrichtungen des Maßregelvollzugs nach $\mathbb{S} \int 63$ und 64 StGB zu ermöglichen, allerdings in Abstimmung mit der aufnehmenden Einrichtung, unter der Voraussetzung einer psychischen Störung und nach Formulierung konkreter Therapieziele, mit der Möglichkeit der Rückverlegung im Ermessen der aufnehmenden Einrichtung,

- die Fragestellung an die Begutachtung vor Anordnung und Verlängerung der Sicherungsverwahrung anzupassen und zu präzisieren mit der Beurteilung, in welchen der bis dahin zu schaffenden Einrichtungen mit unterschiedlichen Sicherungs- und Betreuungsstufen der Betroffene geführt werden kann,

die Konsequenzen der nach den Urteilen des EGMR und des BVerfG erlassenen Regelungen auf das Bewährungsverhalten zu evaluieren.

\section{Die Auswirkung der Regelungen auf die Psychiatrie}

Aus psychiatrischer Sicht sind die neu eingeführte Kategorie einer „psychischen Störung “, deren Konnexität mit Gefährlichkeit sowie die Unterbringung zum Zwecke der „Therapierung “ besonderes problematisch. Hierauf wird im Folgenden eingegangen:

\section{Was ist eine „psychische Störung“?}

Die Europäische Menschenrechtskommission (EMRK) kennt den Begriff des „unsound mind" als eine Voraussetzung einer Freiheitsentziehung. Das Therapieunterbringungsgesetz und später auch das Bundesverfassungsgericht beziehen sich auf die EMRK, wenn sie eine „psychische Störung“ als Voraussetzung einer Unterbringung oder der Verlängerung einer Unterbringung in der Sicherungsverwahrung für Altfälle vorgeben. Mit Hilfe des im ThUG eingeführten Begriffs der „psychischen Störung“ sollen angesichts der EGMR-Rechtsprechung ansonsten zu entlassende, aber weiterhin für gefährlich gehaltene Straftäter weiterhin verwahrt werden können.

Das Therapieunterbringungsgesetz formuliert hierzu den im Rechtssinne bislang undefinierten Begriff der „psychischen Störung “ als Voraussetzung einer Unterbringung und stellt das gültige zweigleisige Strafrechtssystem in Frage: Bislang wurde unterschieden zwischen Straftätern einerseits, die keine krankheitswerte psychische Störung haben und damit für ihre Straftaten verantwortlich sind, und Straftätern andererseits, deren Straftaten direkte Folge ihrer schweren, krankheitswertigen psychischen Störung sind und die daher einen Anspruch auf die Behandlung in der forensischen Psychiatrie haben. Letztere sind vom Gericht als erheblich vermindert schuldfähig oder schuldunfähig eingestuft worden, weil sie krankheitsbedingt nicht oder nur eingeschränkt für ihre Taten verantwortlich waren. Alleine die Feststellung einer psychischen Störung gemäß den aktuellen Klassifikationssystemen ist aber nicht zur Annahme der Schuldminderung oder gar -aufhebung geeignet. Die dort aufgelisteten psychischen Störungen haben höchst unterschiedlichen Einfluss auf die psychosoziale Leistungsfähigkeit und insbesondere auf die Verantwortlichkeit der Betroffenen. Für prosoziale und gemeinschaftsdienliche wie auch für dissoziale und gefährliche Handlungen, die nicht einer krankheitswertigen Störung entspringen, sind deren Täter verantwortlich. Diese verantwortlichen und dennoch gefährlichen Täter sind die Zielgruppe des Therapieunterbringungsgesetzes. Das ThUG ignoriert die unterschiedliche Verhaltensrelevanz der in den Klassifikationssystemen definierten psychischen Störungen, spricht ganz allgemein von psychischer Gestörtheit und macht das Gesetz daher prinzipiell für jedwede (u.a. auch politische) Normabweichung anwendbar. Damit werden abweichendes Verhalten, Gefährlichkeit und Kriminalität in unzulässiger Weise auf psychische Gestörtheit zurückgeführt und letztlich zur Aufgabe der Psychiatrie erklärt.

Mit der Einführung der Kategorie „psychische Störung“ im ThUG wird das Ziel verfolgt, die angesichts der EGMR-Rechtsprechung zu entlassenden, aber weiterhin für gefährlich gehaltenen Straftäter weiterhin verwahren zu können. Damit werden 
gefährliche Straftäter mit einer nicht näher bestimmten „psychischen Störung“ umetikettiert, mithin werden Psychiatrie und die psychiatrischen Diagnosen missbraucht (Müller et al. 2011a). Die Internationalen Klassifikationen psychischer Störungen ICD 10 und DSM IV wurden für die Kommunikation zwischen Medizinern und zwischen Medizinern und Kostenträgern entwickelt. Die darin enthaltenen Diagnosen stehen nicht zur Verwendung für juristische Belange zur Verfügung. Im Zusammenhang mit der Sicherungsverwahrung suggeriert dieser Begriff, dass psychische Störungen mit erheblicher Gefährlichkeit verbunden sind. Dies trifft aber für fast alle Patienten, die die Kriterien einer solchen psychischen Störung erfüllen, gerade nicht zu. Außerdem umfasst der Begriff der „psychischen Störung “ vielfältige und mit unterschiedlichsten Auswirkungen auf die Betroffenen verbundene Störungen von z.B. der ICD-10 Ziffer F00: Demenz bei Alzheimer Erkrankung bis hin zu F99: Nicht näher bezeichnete psychische Störung. Dadurch wird das im ThUG verwandte Eingangsmerkmal so unscharf, dass angesichts der hohen Prävalenzraten für z.B. depressive Störungen, aktuell 3 Millionen Menschen in Deutschland von diesem unscharfen Eingangsmerkmal erfasst würden. An einem bestimmten Zeitpunkt erfüllen etwa 10-20\% der Bevölkerung die Kriterien einer psychischen Störung.

Da die Prävalenzzahlen von Patientinnen und Patienten mit psychischen Störungen in Haftpopulationen noch weitaus höher liegen, würden letztlich über $70 \%$ der Strafgefangenen von diesem Kriterium erfasst (hierzu auch Huchzermeier 2012). Diese Zahlen sollten deutlich machen, dass eine entweder quantifizierende oder anderweitig präzisierende Formulierung erforderlich ist, um die Zielklientel treffend zu beschreiben. In der psychiatrischen Versorgung bewährte Begriffe werden beschädigt, wenn sie - aus dem originären, konzeptionellen Kontext gerissen - Bestandteil von Gesetztexten werden. Deren Nutzung für (straf-) rechtliche Sanktionen stigmatisiert psychiatrische Patienten. Darüber hinaus wird durch die regelhaft notwendigen wissenschaftlichen Überarbeitungen der psychiatrischen Klassifikationen die Intention des Gesetzgebers nach einer beständigen Kategorie verfehlt. Nicht zuletzt deshalb werden die persönliche Eigenschaften erfassenden Merkmale der Gesetzestexte von den psychiatrischen Begriffen unterschieden. Ansonsten werden nämlich Begriffe zu manipulierbaren Hohlformeln. Es ist auch nicht Gegenstand psychiatrischer Expertise einen juristischen Begriff für eine neue, in sicherheitspolitischer Intention zu beschreibende Personengruppe zu definieren; dennoch wurden seitens der DGPPN aus psychiatrischer Sicht weniger schädliche Alternativen zum Begriff „psychische Störung“ vorgeschlagen: in Anlehnung an etablierte juristische Merkmale könnten beispielsweise Begriffe wie „seelische Abartigkeit“, „psychische Abnormität“ bzw. die Kategorien des psychisch abnormen Rechtsbrechers, des sozial desintegrierten Straftäters, des psychisch gestörten Aggressions- oder Sexualstraftäters diskutiert werden, um die zu definierende Personengruppe zu benennen.

Aus psychiatrischer Sicht ist davor zu warnen, das etablierte Störungskonzept der ICD 10 bzw. DSM IV weiter auszudehnen, wie es sich in der Entscheidung des Bundesverfassungsgerichts vom 15.09.2011 (2 BvR 1516/11) andeutet. Wenn der Schweregrad der psychischen Symptomatik nicht einmal die niedrigen Hürden der Kategorie einer psychischen Störung im Sinne der operationalen Diagnosemanuale überschreitet, wird der Boden einer wissenschaftlichen Diagnosestellung verlassen. Ungeachtet dessen wird eine solch vage Beeinträchtigung auch kaum den Kriterien des bislang sehr eng formulierten Konzepts des „unsound mind“ genügen können.

Aus forensisch psychiatrischer Perspektive ist eine „psychische Störung“, die eine Freiheitsentziehung nach dem ThUG rechtfertigt, an der etablierten Auslegung der Schuldfähigkeitsbeurteilung zu orientieren. Diese Kriterien würden allerdings nur bei einer Minderheit der Zielgruppe einer Unterbringung nach dem ThUG erfüllt. Dementsprechend wurde eine deutlich weitere Auslegung des Begriffs „psychische Störung“ gefordert. Doch selbst wenn die weiten Kriterien der operationalisierten Diagnosemanuale zu Grunde gelegt werden, erfüllen nicht alle der Betroffenen die Kriterien einer psychischen Störung. In einer eigenen Studie zur Legalbewährung nach nicht angeordneter nachträglicher Sicherungsverwahrung (Müller et al. 2011 d; Müller et al. 2012), wurden auch die in den ausgewerteten Gutachten gestellten Diagnosen erfasst (Müller et al. 2011d; Tabelle 3). Ungeachtet der auch hier bestätigten hohen und in Übereinstimmung mit weiteren Publikationen zur Prävalenz psychischer Störungen bei Probanden in der Sicherungsverwahrung stehenden Daten (hierzu Habermeyer et al. 2012), hatten 4 der 25 Probanden keine nach den gängigen Diagnosemanualen ICD 10 und DSM IV fassbare Störung, bei weiteren 3 Probanden wurde als psychiatrische Diagnose lediglich eine Substanzmittelabhängigkeit angegeben. Mithin waren bei $28 \%$ der Probanden keine bzw. lediglich eine Substanzmittelabhängigkeit als psychiatrische Diagnose festgehalten. Bei weiteren 6 Probanden wurden zwar auffällige Persönlichkeitszüge beschrieben, eine relevante Persönlichkeitsstörung wurde allerdings nicht festgehalten. Auch bei den Probanden mit einem schwersten Rückfalldelikt, das ist im Sinne der Studiendefinition eine Straftat, die zur Sicherungsverwahrung bzw. der Unterbringung in der psychiatrischen Klinik führte, war nur bei zweien von dreien eine psychiatrische Diagnose festgestellt worden. Dies bedeutet, dass auch in der Gruppe der in dieser Studie binnen 2 Jahren mit schwersten Straftaten rückfällig gewordenen Probanden ein Drittel das Kriterium „psychische Störung“ nicht erfüllt hätten (Müller at al. 2011d).

\section{Konnexität}

Das ThUG formuliert, dass die Gefährlichkeit „infolge“ der „psychischen Störung“ bestehen muss. Die Ausgestaltung der Verknüpfung indes ist streitig. Das BVerfG verlangt im Beschluss vom 04.05.2011 lediglich kumulativ das Vorliegen einer „psychischen Störung “. Allein der Verweis auf das ThUG macht die Notwendigkeit eines Zusammenhangs von „psychischer Störung“ und Gefährlichkeit deutlich (Schöch 2012). Diese Konnexität muss sich nicht notwendig auf die Anlasstat beziehen, könnte aber helfen, die über die „psychische Störung“ definierte Klientel einzugrenzen (Höffler und Stadtland, 2012).

Aus psychiatrischer Sicht ist eine kausale Beziehung zwischen Störung und Gefährlichkeit zu fordern. Allerdings bleibt offen, wie diese Kausalität zu fassen ist. Naheliegend wäre eine kausale Verknüpfung, wenn eine psychische Störung die Einsichts- oder Steuerungsfähigkeit bei Begehung einer Tat erheblich beeinträchtigt hat und insofern die Verantwortlichkeit eingeschränkt oder gar aufgehoben war. Der Schweregrad der Beeinträchtigung müsste bei weiteren $\mathrm{zu}$ erwartenden rechtswidrigen Taten wiederum so ausgeprägt sein, dass die Schuldfähigkeit in erheblicher Weise beeinträchtigt wäre. Diese Klientel - mit eingeschränkter oder aufgehobener Verantwortlichkeit an den weiteren zu befüchtenden Taten - ist aber gerade nicht Zielgruppe des ThUG. 
Dort wird eine nicht verantwortungsrelevante Störung durch ,infolge“ mit Gefährlichkeit verbunden. In diesem Konzept tritt eine die Tat begründende aber nicht verantwortungsrelevante Beeinträchtigung neben die volle Verantwortlichkeit des Täters zum Zeitpunkt seiner Tat. Dies ist widersinnig und widerspricht unserem Verständnis von Verantwortlichkeit und deren Einschränkung. Allenfalls könnte man einen korrelativen Zusammenhang diskutieren, in der Form, dass die Störung mit einer erhöhten Wahrscheinlichkeit weiterer rechtswidriger Handlungen korreliert. Dies wäre aber kein inhaltlicher, kein kausaler Zusammenhang, lediglich ein statistischer Zusammenhang, der auch erst in einem weiteren Schritt auf die Ebene des Individuums bezogen werden müsste. Eine im Zustand voller Verantwortlichkeit begangene Handlung kausal auf eine nicht verantwortungsrelevante Störung zurückzuführen, erscheint hochproblematisch und in sich widersprüchlich. Dies berührt grundsätzlich unser Verständnis vom Verhältnis zwischen Verantwortlichkeit und Persönlichkeitseigenschaften.

\section{Gefährlichkeit}

Neben der „psychischen Störung“ ist die Gefährlichkeit eine weitere Voraussetzung der Unterbringung nach dem ThUG. In Folge der Urteile des EGMR sowie des Bundesverfassungsgerichts ist inzwischen eine Vielzahl Sicherungsverwahrter in Freiheit gesetzt worden. Übergriffe oder kriminelle Handlungen sind bislang jedoch nur in Einzelfällen bekannt geworden. Dies wirft die Frage auf nach der tatsächlichen Gefährlichkeit dieser für hoch gefährlich erachteten Verwahrten. Im forensisch psychiatrischen Kontext ist dies die Frage nach den sogenannten „Falsch Positiven.“ Bei jeder Prognosebeurteilung zur Gefährlichkeit wird vorhergesagt, ob der Proband wieder straffällig werden wird. Diese Prognosen können richtig sein, beispielsweise in der Form, dass Straftaten vorhergesagt werden und der Proband auch wirklich straffällig wird (richtig positiv), oder in der Form, dass keine weiteren Straftaten vorausgesagt werden und der Proband auch wirklich nicht straffällig wird (richtig negativ). Die Prognosen können aber auch falsch sein, beispielsweise in der Form, dass keine weiteren Straftaten vorausgesagt werden, der Proband aber tatsächlich doch straffällig wird (falsch negativ), oder aber dass weitere Straftaten vorausgesagt werden, der Proband aber tatsächlich keine Straftaten mehr begeht bzw. bei Entlassung begangen hätte (falsch positiv). Die falsch negativen Prognosen, also die erneute Straffälligkeit des Probanden bei verneinter Gefährlichkeit, werden im Allgemeinen schnell bekannt und dann in der Regel öffentlich aufgegriffen, schließlich wurden die betroffenen Probanden aufgrund der unzutreffenden günstigen Prognose entlassen oder erhielten Lockerungen und haben damit die Freiheit zu neuen Straftaten genutzt. Dagegen gehen die falsch positiven Beurteilungen zu Lasten des Betroffenen und fordern diesem ein sogenanntes individuelles Sonderopfer für die Gesellschaft ab: Es wird nämlich angenommen, dass der Proband gefährlich ist und erneut Straftaten begehen wird, obwohl er tatsächlich keine Straftaten mehr begangen hätte. Da die Vorhersage, er sei gefährlich, in der Regel zu einer Fortdauer der Inhaftierung oder Unterbringung führt, erhält er keine Gelegenheit, das Gegenteil zu beweisen. Dementsprechend ist zu erwarten, dass eine unbekannt große Anzahl von Betroffenen zu Unrecht dieses Sonderopfer erbringt und inhaftiert beziehungsweise untergebracht wird. Dieses grundsätzlich bekannte Problem wird durch die gegenwärtigen Neuregelungen der Sicherungsverwahrung aktualisiert und wirft die Frage auf, wie gefährlich die Betroffenen wirklich wären, sofern man sie entließe. Es gibt hierzu aus naheliegenden ethischen Gründen keine validen Untersuchungen, schließlich kann man jemanden, von dem man annimmt, er werde wieder mit erheblichen Gewalt- und Sexualdelikten rückfällig, nicht entlassen, um diese Annahme zu überprüfen. Lediglich in wenigen auserlesenen Fällen sind solche Entlassungen auf Grund richterlicher Entscheidungen angeordnet worden und gaben Anhaltspunkte für die tatsächliche Gefährlichkeit der für gefährlich gehaltenen Entlassenen. Damit war die Evaluierung dieser sogenannten natürlichen Experimente möglich:

So klagte Johnnie K. Baxtrom vor dem obersten Bundesgericht der USA gegen seine Unterbringung in einer psychiatrischen Klinik über das Ende seiner Haftzeit hinaus. Das Gericht beurteilte Baxtroms Unterbringung in dieser Spezialeinrichtung für Strafgefangene, die während der Haft psychisch erkrankten, als verfassungswidrig. Daraufhin wurden 1966 insgesamt 967 psychisch kranke Rechtsbrecher aus besonders gesicherten Einrichtungen in eine von 18 allgemeinpsychiatrischen Kliniken verlegt. Viele wurden dort bald entlassen. Nach maximal 4 Jahren wurden 199 Patienten zufällig ausgewählt und nachuntersucht. 14 waren erneut mit gewalttätigem Verhalten aufgefallen, 44 Patienten wurden wieder untergebracht. Die überwiegende Mehrheit aber war straffrei geblieben oder ist nur noch mit Bagatelldelikten in Erscheinung getreten (Steadman und Cocozza 1974).

1971 klagte der Amerikaner Dixon wegen mangelhafter Unterbringungsbedingungen vor einem Bezirksgericht in Pennsylvania. Infolge des Urteils wurden von den $561 \mathrm{~Pa}-$ tienten der Hochsicherheitseinrichtung 389 Patienten entlassen. Im Nachbeobachtungszeitraum von maximal 4,5 Jahren wurden 23,7 \% erneut festgenommen (Thornberry und Jacoby 1979).

Mit Ende der DDR wurden 32 Patienten, die in der ehemaligen DDR verurteilt und nach der Wiedervereinigung wegen ihrer besonderen Gefährlichkeit im Maßregelvollzug der neuen Bundesländer untergebracht worden waren, 1994 durch eine Entscheidung des Bundesverfassungsgerichtes trotz schlechter Prognose entlassen. Nach etwa 7 Jahren waren nur 5 von ihnen mit gefährlichen Straftaten rückfällig geworden. Die Mehrheit der entlassenen 32 Patienten war in zum Teil geschlossenen Heimen und Übergangswohnheimen untergebracht worden (Stadtland 2006).

Eine vergleichbare Situation entstand 2004, als die Möglichkeit der nachträglichen Unterbringung in der Sicherungsverwahrung ( $\$ 66 \mathrm{~b}$ StGB) geschaffen wurde. Dies wurde in der oben bereits erwähnten Studie aufgegriffen (Müller et al. 2011d). Seit 29.07.2004 ist die nachträgliche Unterbringung in der Sicherungsverwahrung (nSV) möglich. Diese Unterbringung ist keine Revision des Indexurteils, sondern erfordert sogenannte „Nova“, also neue Anknüpfungstatsachen, die bei der Indexverurteilung nicht bekannt waren und nachträglich zu einer veränderten Einschätzung der Gefährlichkeit des Probanden führen. Die Einführung der nSV war jederzeit umstritten. Insbesondere die Streitigkeit der sogenannten „Nova“ führte dazu, dass die für erforderlich erachtete Verhängung der nSV vom Bundesgerichtshof in der Mehrzahl der Fälle aufgehoben und letztlich die betroffenen Probanden in Freiheit entlassen wurden. Dies ermöglicht es, die Legalbewährung der für gefährlich gehaltenen Entlassenen zu untersuchen. In dem untersuchten Bewährungszeitraum von mindestens 24 Monaten in Freiheit wurden 7 von 25 Probanden $(28 \%)$ mit schweren Straftaten rückfällig (Tabelle 1). $32 \%$ wurden mit Rechtsverstößen auffällig, die mit Geldstrafe oder 
Haft von nicht über 6 Monaten geahndet wurden und die im Kontext der Sicherungsverwahrung als Bagatelldelikte bezeichnet werden können (Tabelle 2a). Dies bedeutet, dass nach diesen Studienkriterien und unter Beachtung der unten genannten Grenzen etwa $60 \%$ der Probanden als so gefährlich angesehen wurden, dass sie über die Verbüßung ihrer Haftstrafe hinaus in Verwahrung genommen werden sollten, obwohl sie tatsächlich in Freiheit nicht mit den geforderten schweren Delikten rückfällig wurden (Tabelle 2b). Dabei wurde als schweres Rückfalldelikt gewertet, wenn in dem Folgeurteil mindestens eine einjährige Haftstrafe ohne Bewährung verhängt worden war. Dieses Kriterium als Maßstab für die Notwendigkeit der Anordnung der Sicherungsverwahrung ist unter Umständen sogar ein zu weiches. Angesichts der bei Wiederholungsdelinquenz zunehmenden Strafhärte sind zeitige Freiheitsstrafen möglicherweise nicht das diskriminierende Kriterium für die zu beschreibende Gefährlichkeit. Stattdessen müssten sehr langfristige Haftstrafen oder besser noch die zeitlich nicht befristete Unterbringung in einem psychiatrischen Krankenhaus beziehungsweise natürlich die in der Folgeentscheidung angeordnete Sicherungsverwahrung als relevante Zielkriterien diskutiert werden. In der vorliegenden Klientel wurde die Sicherungsverwahrung in zwei Fällen, die Unterbringung im psychiatrischen Krankenhaus in einem Fall verhängt. Dementsprechend finden sich in der vorliegenden Untersuchungsklientel in 3 von 25 Fällen, also bei $12 \%$, schwerste Delikte, die mit einer Sicherungsverwahrung beziehungsweise mit unbefristeter Unterbringung in einem psychiatrischen Krankenhaus sanktioniert wurden (Tabelle 2b). Ob dieser Anteil, und insbesondere unter Einbeziehung der schweren, mit einer Haftstrafe geahndeten Delikte, hinreichend gravierend ist, den übrigen $88 \%$ beziehungsweise 72 $\%$ das individuelle Sonderopfer für die gesellschaftliche Sicherheit abzuverlangen, ist politisch zu diskutieren und gesellschaftlich zu verantworten. Diese Zahlen hinsichtlich der Legalbewährung gehen nicht nur weitgehend mit den Ergebnissen der eingangs skizzierten natürlichen Experimente konform, sie entsprechen auch den anderen bislang vorgelegten Studien zur Rückfälligkeit bei Sicherungsverwahrten (Alex 2010; Kinzig 2011).

\section{Therapie(rung)}

Die Maßregel der Sicherungsverwahrung ist die einschneidendste Sanktion des deutschen Strafrechts, die von den Betroffenen ein großes individuelles Sonderopfer abverlangt. Das Bundesverfassungsgericht hat mit Urteil vom 04.05.2011 den Ausnahmecharakter dieser Maßregel erneut deutlich gemacht und deren Intention auf die Verhinderung schwerster Straftaten eingeengt. Das Bundesverfassungsgericht hebt hervor, dass das Sonderopfer, das von den Verwahrten erbracht werden muss, besondere Rahmenbedingungen erfordert. Grundsätzliche Bedenken, die aufgeworfen werden, wenn eine therapeutisch besonders problematische Klientel zum Zwecke der Therapierung verwahrt werden soll und hierzu bislang keine geeigneten Therapieansätze erprobt sind, wurden seitens der DGPPN formuliert (Müller et al. 2011a). Insbesondere wurde von einer Diskreditierung des Therapiebegriffs gewarnt und darauf hingewiesen, dass es für einen durchzuführenden Therapieansatz sicher nicht hinreichend sein kann, wenn lediglich dessen Wirkungslosigkeit nicht bewiesen ist. Dennoch wurden konstruktive Empfehlungen für die Ausgestaltung der Sicherungsverwahrung gegeben (Müller et al. 2011c). So sind die Unterbringungsbedingungen in der Sicherungsverwahrung deutlich von Inhaftierungsbedingungen abzuheben und die Verwahrten durch spezielle, auch individualisierte Therapieangebote frühzeitig, bereits während des Strafvollzugs, an eine Entlassungsvorbereitung heranzuführen. Diese Therapieund Lockerungsmaßnahmen müssen die individuelle Gefährlichkeit des Verwahrten ebenso wie die Behandelbarkeit und Prognose einer bei ihm diagnostizierten Störung berücksichtigen und setzen in der Therapie mit Untergebrachten und Inhaftierten erfahrene Therapeuten voraus. Dies erfordert eine erkennbare Ausrichtung der Sicherungsverwahrung auf Therapie und Entlassungsvorbereitung, die notwendig eine deutliche Anpassung des Therapeutenschlüssels und eine therapieorientierte Umgestaltung der Einrichtungen nach sich ziehen muss. Hierzu werden in manchen Bundesländern bereits die SothAs angepasst, erweitert und therapieorientiert umgestaltet. Trotz einer solchen Umgestaltung der SothAs kann in Einzelfällen dennoch eine Verlegung aus den Haftanstalten und den SothAs in Einrichtungen des Maßregelvollzugs nach $\int \mathbb{S} 63$ oder 64 StGB sinnvoll und zielführend sein. Die Verlegung der Betroffen muss dabei an eine konkrete Indikation zur psychiatrisch- psychotherapeutischen Behandlung einer diagnostizierten Störung gebunden sein. Zusätzlich zur festgestellten Diagnose sind auch konkrete Therapieziele zu formulieren. Das aufnehmende Krankenhaus ist hierbei einzubeziehen und soll der Verlegung zustimmen. Nach Abschluss der Therapie, bei Wegfall der Grundlage der Verlegung und wenn sich ein Therapieziel als nicht erreichbar erweist, muss die aufnehmende Einrichtung die Möglichkeit haben, den Betroffenen nach Ermessen zurückzuverlegen. Dieses Vorgehen impliziert eine grundsätzliche Öffnung der Maßregelvollzugskrankenhäuser für Straftäter mit einer psychischen Störung, ohne den besonderen therapeutischen Charakter der psychiatrischen Kliniken zu zerstören (hierzu: Huchzermeier 2012).

Bislang sieht das Konzept des Bundesministeriums für Justiz jedoch keine differenzierte Abstufung der Unterbringungs- und Betreuungskonzepte für Sicherungsverwahrte vor. Diese sind angesichts des Resozialisierungsauftrags aber unerlässlich und sollten in Anlehnung an die im psychiatrischen Maßregelvollzug bewährten Behandlungs- und Lockerungskonzepte stufengliedrig erfolgen, beispielweise:

1) Geschlossene Einrichtungen mit der Möglichkeit zur intramuralen Teilnahme an intensiven Betreuungsangeboten durch psychiatrisch-psychotherapeutisch ausgebildetes Personal.

2) Halboffene bzw. weniger gesicherte Übergangsstationen mit der Vorbereitung und Förderung externer Aktivitäten.

3) Angegliederte resozialisierungsorientierte Einrichtungen mit multiprofessioneller Betreuung und unterstützenden psychotherapeutischen Angeboten.

4) Sozio- und psychotherapeutisch ausgerichtete Nachsorgeambulanzen.

5) Ungeachtet eines solchen Betreuungsmodells bleiben allerdings Abteilungen für nicht behandelbare, nicht veränderungsbereite und weiterhin gefährliche Delinquenten unverzichtbar.

Diese Strategie des gestuften Risikomanagements mit langfristigen Erprobungen in kontrollierten Lockerungen und ambulanter Nachsorge ist geeignet, die individuelle Resozialisierung zu fördern und zugleich ein ausgewogenes Maß von Sicherungsmaßnahmen und den Schutz der Öffentlichkeit herzustellen. Die Entwicklung des Unter- 
gebrachten, dessen Scheitern in einer konkreten Erprobungsstufe mit der Möglichkeit der Rückstufung, beziehungsweise dessen Bewährung unter den gegebenen Bedingungen mit der Möglichkeit weiterer Lockerungen und Erprobungen, sind anlassbezogen zu beurteilen. Die grundsätzliche Möglichkeit auch niederschwelligerer Betreuung zeigen die Verläufe der in Folge der Urteile des EGMR und des Urteils des Bundesverfassungsgerichts inzwischen aus der Unterbringung entlassenen und anderen Betreuungskonzepten zugeführten Verwahrten. Bislang sind nur vereinzelte Rückfälle bekannt geworden. Das Berliner Modell der ambulanten Betreuung berichtet nach einem Jahr von einem schwerwiegenden Rückfalldelikt entsprechend eines Anteils von unter $10 \%$. Dies verdeutlicht die grundsätzliche Möglichkeit alternativer, auch extramuraler Betreuungskonzepte und illustriert deren Stellenwert im Rahmen eines gestuften Betreuungssettings (Voß et al. 2012).

Darüber hinaus regt das Bundesverfassungsgericht mit dem Urteil vom 04.05.2011 an, individuelle Behandlungskonzepte zu entwickeln, wenn sich die etablierten Verfahren als nicht wirkungsvoll erweisen. Ähnlich ermuntert auch das ThUG zu Behandlungsversuchen, wenn es eine Behandlung dann bereits als geeignet ansieht, wenn deren Wirkungslosigkeit nicht belegt ist. Damit wird ein weites Feld eröffnet, neue Behandlungsverfahren anzuwenden und zu erproben. Die Behandlungsverfahren, die bei Menschen eingesetzt werden, müssen nun aber hinsichtlich der Nebenwirkungen, ihres Nutzen und ihrer Verlässlichkeit bekannt sein. Dies ist nur im Rahmen eines wissenschaftlichen Vorgehens mit einer entsprechenden Behandlungsevaluation ethisch vertretbar. Dies wäre aber Forschung an Untergebrachten und damit ethisch und juristisch höchst brisant (Duttge 2012). Insofern setzen höchstrichterliche Rechtsprechung wie auch Gesetzgeber und deren Hoffnung auf Behandlung und Behandelbarkeit hoch gefährlicher Straftäter neue Impulse für Wissenschaft und Forschung im Kontext forensisch relevanter psychischer Störungen.

\section{Resumee}

Aus der aktuellen Entscheidung des BVerfG, dem ThUG und aktuellen Gesetzentwürfen ergeben sich schwerwiegende Implikationen für die Psychiatrie, die in den Stellungsnahmen der DGPPN aus dem Jahre 2011 (Müller et al. $2011 \mathrm{a}-\mathrm{c}$ ) dargestellt wurden. Insbesondere die Bestimmungen des ThUG zur psychischen Störung als Unterbringungsvoraussetzung sowie zur „Therapierung“ wurden als Missbrauch der Psychiatrie bezeichnet und illustrieren die zeitlose Gefährdung der Psychiatrie, für politische Zwecke funktionalisiert zu werden. Nahezu regelhaft nämlich gerät die Psychiatrie ins Visier, wenn die Gesellschaft noch besser vor gefährlichen Straftätern geschützt werden soll. Die historische Dimension der Problematik illustriert der Münchner Psychiater und Schriftsteller Oskar Panizza:

„(...) das Prinzip der Humanität, welches bei unseren heutigen, auf allen Gebieten aufgeregten Zeiten, besonders auch im Gerichtssaal immer wieder an die Spize gestellt wird, muss einer Krankheitsform die höchste Beachtung zuwenden, die, wenn richtig erkant und angewant, eine grosse Zahl von dem Gefängnis und Zuchthaus verfallener Individuen in die milderen Räume und freundlichen Badewannen der Irrenhäuser hinüberführt. Und bei dieser Translozirung mitgewirkt zu haben, ist wahrlich auch des Schweisses edler Psichjater wert. (...) Ein mässig grosses Irrenhaus zwischen Nekar und Rhein, etwa von der Grösse der Pfalz und auf eben diesen Boden, wo die turbulentesten Köpfe gediehen, errichtet,

Tab. 1a

Rückfälligkeit bei abgelehnter nachträglicher Sicherungsverwahrung (Wie gefährlich sind die Kandidaten für die nachträgliche SV?)

\begin{tabular}{|l|l|l|}
\hline Gruppe & Anzahl der Pbn & $\begin{array}{l}\text { Time at risk } \\
(\text { MW in Mon. })\end{array}$ \\
\hline Kein Rückfall & $\begin{array}{l}10 \\
(40 \%)\end{array}$ & 26,8 \\
\hline Leichter Rückfall & $\begin{array}{l}8 \\
(32 \%)\end{array}$ & 13,4 \\
\hline Schwerer Rückfall & $\begin{array}{l}7 \\
(28 \%)\end{array}$ & 11,1 \\
\hline
\end{tabular}

Tab.2a : Delikte bei Rückfällen nach abgelehnter nSV Gruppe: leichter Rückfall

$\mathrm{N}=25, \mathrm{n}=8$

\begin{tabular}{|l|l|l|}
\hline Indexdelikt & Rückfall-Delikt & Strafmaß \\
\hline Schwerer Raub & Falsche uneidl.Aussage & Geldstrafe \\
\hline Vergewaltigung & Verstoß gg FA-Weisungen & Geldstrafe \\
\hline Mord in 2 Fällen & BtmG-Verstoß & FS 4 Monate \\
\hline $\begin{array}{l}\text { Schwerer sex. Mißbrauch, } \\
\text { Kinderpornographie }\end{array}$ & Fahrlässige Trunkenheit & $\begin{array}{l}\text { Geldstrafe; Sperre Fahr- } \\
\text { erlaubnis }\end{array}$ \\
\hline $\begin{array}{l}\text { Schwerer Menschenhandel, } \\
\text { Zuhälterei }\end{array}$ & $\begin{array}{l}\text { 1. Leistungserschleichung; } \\
\text { 2. Verstoß gg FA-Weisungen }\end{array}$ & $\begin{array}{l}\text { Geldstrafe; } \\
\text { \$63 StGB z.Bewährung }\end{array}$ \\
\hline Sex. Mißbrauch & Diebstahl geringw. Sachen & Geldstrafe \\
\hline Sex. Mißbrauch & Betrug & Geldstrafe \\
\hline Vers. Totschlag & Verstoß gg FA-Weisung & FS 3 Mon. z. Bewährung \\
\hline
\end{tabular}

hätte über Nacht die kriminelle Bewegung, ich wolte sagen: die epidemische Psichose, im Keime erstikt und unserem Vaterlande viel Leids erspart“ (Panizza, 1898).

Die Satire Panizzas illustriert die zeitlose Gefährdung der Psychiatrie, für politische Zwecke funktionalisiert zu werden. Auch als um die Jahrtausendwende die Frage aufgeworfen wurde, was mit gesunden, doch weiterhin potentiell gefährlichen Straftätern zu tun sei, die nach der Haftentlassung ein mehr oder weniger großes Sicherheitsrisiko bargen, wurde in mehreren Bundesländern versucht, diese Straftäter mit Hilfe der jeweiligen Unterbringungsgesetze dauerhaft in der Psychiatrie unterzubringen, indem Indikationsbereich und Krankheitsbegriff erheblich ausgeweitet wurden. Dem trat das Bayerische Oberste Landesgericht im Beschluss vom 17.12.2001 (3ZR386/01) entgegen (Müller et al., 2002). 10 Jahre später wird mit Hilfe des Konzeptes einer „psychischen Störung “ und therapeutischen Mitteln erneut Kriminalprävention betrieben. Dem wurde seitens der psychiatrischen Fachgesellschaft energisch entgegengetreten. 
Tab. 2b: Delikte bei Rückfällen nach abgelehnter nSV Gruppe: einschl./schwerer Rückfall

$\mathrm{N}=25, \mathrm{n}=7$

Kursiv gedruckt: Probanden mit schwersten Delikten, d.h. mit Unterbringung gemäß $\$ 63$ StGB bzw. $\$ 66$ StGB geahndeten Delikten

\begin{tabular}{|l|l|l|}
\hline Indexdelikt & Delikt & Strafmaß \\
\hline $\begin{array}{l}\text { Schwere Brandstiftung in TE } \\
\text { mit Körperverletzung }\end{array}$ & $\begin{array}{l}\text { Vers. Schwere Brandstiftg. in } \\
\text { TE mit vors KV in TE mit SB }\end{array}$ & 6 J FS + SV \\
\hline $\begin{array}{l}\text { Vers. Räub. Erpressung in TE } \\
\text { mit gef. KV }\end{array}$ & $\begin{array}{l}\text { 1. Raub + vers.Raub; } \\
\text { 2. Btm-Verstoß; } \\
\text { 3. Nachstellung, Beleidigung, } \\
\text { Bedrohung }\end{array}$ & $\begin{array}{l}\text { 2 J 10 Mon FS; } \\
\text { 3 Mon FS; } \\
5 \text { Mon FS }\end{array}$ \\
\hline Vergewaltigung & $\begin{array}{l}\text { Sex. Mißbrauch widerstands- } \\
\text { unfähger Personen }\end{array}$ & S63 StGB \\
\hline Vergewaltigung & $\begin{array}{l}\text { KV + Diebstahl, KV in TE mit } \\
\text { Nötigung, gems. Raub in TE } \\
\text { mit KV }\end{array}$ & 2 J Mon FS \\
\hline $\begin{array}{l}\text { Vers.Vergew. in TE mit sex. } \\
\text { Nötigung, sex.Mißbrauch }\end{array}$ & $\begin{array}{l}\text { Sex. Nötigung in TE mit } \\
\text { vorsätzl. KV }\end{array}$ & 3 J FS + SV \\
\hline Mord in 2 Fällen & $\begin{array}{l}\text { Vorsätzl. KV 2 Fälle, vers. } \\
\text { Nötigung 2 Fälle, vorsätzl. } \\
\text { Vollrausch }\end{array}$ & 1 J 4 Mon FS \\
\hline Vergewaltigung & $\begin{array}{l}\text { 1. Diebstahl; } \\
\text { 2. Nötigung in TM mit vers. } \\
\text { Diebstahl }\end{array}$ & Geldstrafe; \\
\hline
\end{tabular}

Tab.3: Diagnosen laut Gutachten zur nSV (Mehrfachnennungen möglich)

\begin{tabular}{|r|c|c|c|}
\hline Diagnose & $\begin{array}{c}\text { Kein Rückfall } \\
\text { (Anzahl GA= 22) }\end{array}$ & $\begin{array}{c}\text { Leichter RF } \\
(\text { Anzahl GA= 19) }\end{array}$ & $\begin{array}{c}\text { Schwerer RF } \\
\text { (Anzahl GA= 14) }\end{array}$ \\
\hline $\begin{array}{r}\text { Diss. Persönlichkeits- } \\
\text { störung }\end{array}$ & 8 & 7 & 7 \\
\hline $\begin{array}{r}\text { Keine Persönlichkeits- } \\
\text { störung }\end{array}$ & 8 & 4 & 3 \\
\hline C2/Drogen & 5 & 12 & - \\
\hline Psychose & - & 2 & - \\
\hline Pädophilie & 6 & 4 & - \\
\hline Andere Sex.störg & 3 & - & 12 \\
\hline
\end{tabular}

\section{Literatur}

Alex M. (2010) Nachträgliche Sicherungsverwahrung - ein rechtsstaatliches und kriminalpolitisches Debakel. Bochumer Schriften Bd. 11, Felix Verlag, Holzkirchen.

Dittmann, V. (2011) „Psychische Störung“ im Therapieunterbringungsgesetz (ThUG) und im Urteil des Bundesverfassungsgerichts vom 4. Mai 2011 - Versuch einer Klärung, in: N. Nedopil (Hrsg.), Die Psychiatrie und das Recht - Abgrenzung und Brückenschlag. Jubiläumsschrift zum vierzigjährigen Bestehen der Abteilung für Forensische Psychiatrie der Psychiatrischen Klinik der Universität München. Pabst Science Publishers Lengerich, S. 131-150.

Duttge, G. (2010) Forschung an Untergebrachten, in: Müller J.L. (Hrsg.), Neurobiologie forensisch relevanter Störungen. Grundlagen, Störungsbilder, Perspektiven. Kohlhammer Verlag, S.47-58.

Habermeyer, E. und Vohs, K. Kriminologische und diagnostische Merkmale von Sicherungsverwahrten in: J. Müller, N. Nedopil, N. Saimeh, E. Habermeyer, P. Falkai (Hrsg.), Sicherungsverwahrung - wissenschaftliche Basis und Positionsbestimmung. MWV Berlin 2012 (in press).
Höffler, K. und Stadtland C. Mad or bad? Der Begriff „psychische Störung “ des ThUG im Lichte der Rechtsprechung des BVerfG und des EGMR. Der Strafverteidiger 2012, S. 239.

Huchzermeier, C.H. Persönlichkeitsmerkmale von Straftätern und psychotherapeutische BeJ. Müller, N. Nedopil, N. Saimeh, E. Habermeyer, P. Falkai (Hrsg.), Sicherungsverwahrung - wissenschaftliche Basis und Positionsbestimmung. MWV Berlin 2012 (in press).

Kinzig J. (2011) Empirische Erkenntnisse zur Sicherungsverwahrung als Grundlage der gutachterlichen Beurteilung, in: N. Nedopil (Hrsg.), Die Psychiatrie und das Recht - Abgrenzung und Brückenschlag. Jubiläumsschrift zum vierzigjährigen Bestehen der Abteilung für Forensische Psychiatrie der Psychiatrischen Klinik der Universität München. Pabst Science Publishers Lengerich, S. 78-91.

Koller, M. Was versteht der EGMR unter „unsound mind" (Art. 5 Abs. 1S.2 lit.e EMRK)?, in: J. Müller, N. Nedopil, N. Saimeh, E. Habermeyer, P. Falkai (Hrsg.), Sicherungsverwahrung - wissenschaftliche Basis und Positionsbestimmung. MWV Berlin 2012 (in press). handlungsmöglichkeiten in Haftanstalten, in:
Müller, J. L., Klein, H. E. \& Cording, C. (2002). Missbrauch der Psychiatrie? Konflikte zwischen staatlichem Sicherheitsanspruch und dem Selbstverständnis psychiatrischer Kliniken, in H.-H. Kühne, H. Jung, A. Kreuzer, J. Wolter (Hrsg.), Festschrift für Klaus Rolinski. Baden-Baden: Nomos, S. 447-458.

Müller, J.L., Saimeh, N., Habermeyer, E., Nedopil, N., Schneider, F., Falkai, P. (2011a). Stellungnahme der Deutschen Gesellschaft für Psychiatrie, Psychotherapie und Nervenheilkunde (DGPPN) zum Gesetz zur Neuordnung des Rechts der Sicherungsverwahrung und zu den begleitenden Regelungen, und insbesondere zum Artikel 5 Gesetz zur Therapierung und Unterbringung psychisch gestörter Gewalttäter (Therapieunterbringungsgesetz - ThUG in Kraft seit 01.01.2011 vom 10.02.2011. Nervenarzt 2011, 82: 382-383.

Müller, J.L., Nedopil, N, Saimeh, N., Schneider, F., Falkai, P. (2011b). Stellungnahme der Deutschen Gesellschaft für Psychiatrie, Psychotherapie und Nervenheilkunde (DGPPN). Zur Entscheidung des Bundesverfassungsgerichts zu den Regelungen zur Sicherungsverwahrung vom 04.05.2011 vom 10.06.2011. Nervenarzt 2011, 82: 933-935.

Müller, J.L., Saimeh, N., Habermeyer, E., Nedopil, N., Schneider, F., Falkai, P. (2011c). Empfehlungen der Deutschen Gesellschaft für Psychiatrie, Psychotherapie und Nervenheilkunde (DGPPN) zur Neuregelung der Sicherungsverwahrung angesichts des Urteils des Bundesverfassungsgerichts vom 04.05.2011 vom 11.10.2011. Nervenarzt 2011, 82: 1497-1504.

Müller, J. L., Stolpmann G., Fromberger P., Haase K. A., Jordan K. (2011d) Psychiatrische Gutachten zur nachträglichen Sicherungsverwahrung, Monatsschrift für Kriminologie und Strafrechtsreform 4 (11) S. 253-265.

Müller, J. L., Stolpmann, G. Untersuchung der nicht angeordneten nachträglichen Sicherungsverwahrung - Implikationen für die Neuregelung der Sicherungsverwahrung, in: J. Müller, N. Nedopil, N. Saimeh, E. Habermeyer, P. Falkai (Hrsg.), Sicherungsverwahrung - wissenschaftliche Basis und Positionsbestimmung. MWV Berlin 2012 (in press).

Panizza, O. (1989). Psichopatia criminalis. Anleitung um die vom Gericht für notwendig erkanten Geisteskrankheiten psichjatrisch zu eruieren und wissenschaftlich festzustellen. Zürich. Verlag der Zürcher Diskussionen, in: O. Panizza (1985), Die kriminelle Psychose, genannt Psichopatia criminalis. Hilfsbuch für Ärzte, Laien, Juristen, Vormünder, Verwaltungsbeamte, Minister etc. Zur Diagnose der politischen Gehirnerkrankung. 2. Aufl. München: Matthes \& Seitz.

Pollähne, H. (2011). Sicherung durchgebrannt? Ein ThUG, wer Böses dabei denkt. Recht \& Psychiatrie 1, 2.

Schöch, H. (2012). Das Urteil des Bundesverfassungsgerichts zur Sicherungsverwahrung. Goltdammer's Archiv für Strafrecht (1) 159 2012, 14-31.

Steadman, H., Cocozza, J. (1974) Careers of the Criminally Insane. Lexington. Mass. Lexington Book.

Stadtland, C. (2006) Risikoeinschätzung und Rückfallprognosen in der Forensischen Psychiatrie: Das Münchener Prognoseprojekt (MPP), Habilitationsschrift. München.

Thornberry, T., Jacoby, J. (1979) The criminally insane: A Community follow up of mentally ill offenders. Chicago University of Chicago Press.

Voß, T., Sauter, J., Kröber, H.-L., Ambulante Betreuung von aufgrund des BVG-Urteils entlassener Sicherungsverwahrter in: J. Müller, N. Nedopil, N. Saimeh, E. Habermeyer, P. Falkai (Hrsg.), Sicherungsverwahrung wissenschaftliche Basis und Positionsbestimmung. MWV Berlin 2012 (in press). 